\title{
The Paths of Quintessence
}

\author{
Eric V. Linder \\ Berkeley Lab, University of California, Berkeley, CA 94720
}

(Dated: March 13, 2006)

\begin{abstract}
The structure of the dark energy equation of state phase plane holds important information on the nature of the physics. We explain the bounds of the freezing and thawing models of scalar field dark energy in terms of the tension between the steepness of the potential vs. the Hubble drag. Additionally, we extend the phase plane structure to modified gravity theories, examine trajectories of models with certain properties, and categorize regions in terms of scalar field hierarchical parameters, showing that dark energy is generically not a slow roll phenomenon.
\end{abstract}

\section{INTRODUCTION}

The discovery of the acceleration of the cosmic expansion has thrown physics and astronomy research into a ferment of activity, from a search for fundamental theories to investigation of predictions relating models and the cosmological dynamics, to development of astrophysical surveys yielding improved measurements.

The acceleration, or more generally the expansion history of the scale factor evolution with time, $a(t)$, can be equivalently treated by a dark energy pressure to density, or equation of state, ratio $w(a)$ [1]. One model, Einstein's cosmological constant, predicts $w=-1$ at all times, but generically the dark energy phenomenon has dynamics, a time varying $w(a)$.

It is important to note that the current epoch of accelerated expansion is very different from the early epoch of inflation. We know a priori that dark energy does not completely dominate the universe now and we do not know a priori that dark energy obeys a slow roll approximation (in fact we will see it is unlikely to).

In these senses, dark energy is a more challenging phenomenon than inflation. We are faced with three "Goldilocks" problems: 1) dark energy is dynamically important, but not fully dominant, with $\sim 75 \%$ of the total energy density today, 2) the universe is accelerating, as from a component with $w \lesssim-0.8$, but the field responsible may not be slowly rolling (unless fine tuned) as it would if nearly completely potential dominated, and 3 ) if it's not the cosmological constant, what happened to the cosmological constant?

To discover whether the physics is the cosmological constant, and to distinguish between alternate theories, requires measurement of the possible dynamics.

Caldwell \& Linder [2] (Paper 1) investigated the dynamics in the phase plane of $w-w^{\prime}$, where $w^{\prime}=d w / d \ln a$, for canonical scalar field dark energy, or quintessence, finding that this reveals important clues to the nature of the new physics. In such a plane, the time or scale factor variable is a parameter along the paths of dynamics. They found distinct structure, categorizing fields into those that at early times are locked by Hubble friction into a cosmological constant like state, and then move away from this (thaw) as the dark energy dominates, and those that initially roll due to the steepness of the potential but later approach the cosmological constant (freeze). In this article some of these results are put on a firmer footing, examined in greater detail, and extended to models beyond canonical scalar fields, including modified gravity theories.

\section{GENERAL DYNAMICS}

We begin with a brief, explicit derivation of the key dynamics equation, Eq. (6). A scalar field $\phi$ possesses both potential energy $V(\phi)$ and kinetic energy $(1 / 2) \dot{\phi}^{2}$. The Lagrangian density is of the form

$$
\mathcal{L}=(1 / 2) g_{\mu \nu} \partial^{\mu} \phi \partial^{\nu} \phi-V(\phi),
$$

for a canonical, minimally coupled scalar field in a metric $g_{\mu \nu}$. The equation of motion for the field, the KleinGordon equation,

$$
\ddot{\phi}+3 H \dot{\phi}+V_{, \phi}=0,
$$

where $H=\dot{a} / a$ is the Hubble parameter, follows from functional variation of the Lagrangian. (Spatial inhomogeneities should be negligible on subhorizon scales, but also see $\S \mathrm{VIC}$.) The energy-momentum tensor is generated through Noether's theorem and one can identify the energy density and pressure:

$$
\rho=(1 / 2) \dot{\phi}^{2}+V(\phi) \quad ; \quad p=(1 / 2) \dot{\phi}^{2}-V(\phi) .
$$

It will be convenient to invert these and write the potential and kinetic energies in terms of $\rho$ and $w$ :

$$
V=(1 / 2)(1-w) \rho \quad ; \quad(1 / 2) \dot{\phi}^{2}=(1 / 2)(1+w) \rho .
$$

Note that $w$ and $\rho$ are both functions of time, or scale factor.

To obtain an equation for the variation $w^{\prime}$, take the derivative of $V$ with respect to $\phi$,

$$
V_{, \phi}=\dot{V} / \dot{\phi}=(1 / 2)[(1-w) \dot{\rho}-\rho \dot{w}] /[(1+w) \rho]^{1 / 2} .
$$

Employing the continuity equation $\dot{\rho}=-3 H \rho(1+w)$ and $H=d \ln a / d t$, one obtains

$w^{\prime}=-3\left(1-w^{2}\right)-(1-w)(1+w)^{1 / 2} \sqrt{(3 / 8 \pi) \Omega_{\phi}(a)} \frac{M_{P} V_{, \phi}}{V}$,

where $\Omega_{\phi}(a)=8 \pi \rho /\left(3 H^{2} M_{P}^{2}\right)$ is the dimensionless dark energy density, and $M_{P}$ is the Planck mass. 


\section{A. Distinguishing $\Lambda$}

We see that $w^{\prime}$ is related to the nearness of the equation of state ratio to the cosmological constant value, i.e. $1+w$, and the inverse of the characteristic field scale of the potential, $V_{, \phi} / V$ (sometimes phrased as a slow roll parameter, $\left.M_{P}\left|V_{, \phi} / V\right| \ll 1\right)$. If $w$ is readily distinguished from -1 , then we know the dark energy is not a cosmological constant, regardless of the value of the time variation $w^{\prime}$. More difficult is the case where $\epsilon=1+w$ is a small quantity. Then it will be quite important to determine whether $w^{\prime}$ is zero or not. Eq. (6) guides us in following the dynamics in the $w-w^{\prime}$ phase space.

The first point to notice is that the reciprocal of the characteristic field scale is not generically a small parameter useful for a "slow roll" approximation. Figure 1 shows curves of constant field scale

$$
\Phi=V /\left(-V_{, \phi}\right)
$$

in the $w-w^{\prime}$ plane. Only a tiny sliver of the phase space, plus a small hump, satisfies the slow roll approximation; unless one is exceedingly close to the cosmological constant behavior there is substantial dynamics in the field.

When $1+w \ll 1$, the second term in Eq. (6) dominates and $w^{\prime}>0$ (creating the "humps"), while for less negative $w$ the first term can dominate and drive $w^{\prime}<0$. This driving occurs closer to $w=-1$ for larger $\Phi$. Within the large- $\Phi$ hump, the dark energy looks similar to a cosmological constant. Suppose one conjectures some physics limiting the size of the field scale, equivalently leading to avoidance of flatness in the potential. An upper bound on $\Phi$ in the scalar field behavior would impose a barrier around the cosmological constant $\Lambda$, saying that the scalar field dynamics must be distinguishable from $\Lambda$, if it is not $\Lambda$.

The second panel of Fig. 1 zooms in to illustrate this barrier for $\Phi<M_{P}$, which rules out any freezing field, and any thawing field with $1+w<0.004$. So any scalar field theory with field scales barred from being transPlanckian are distinguishable from $\Lambda$ at this precision. If the restriction uses, say, $\Phi<M_{P} / \sqrt{8 \pi}$ (i.e. the Planck mass is defined in terms of Newton's constant as $G_{N}=\left(8 \pi M_{P}^{2}\right)^{-1}$ rather than $G_{N}=M_{P}^{-2}$ as above) then the limit becomes $1+w>0.1$.

The physical origin for the conjectured limit on the characteristic field scale is not clear, but the implications are important enough to consider the possibility. Since the field scale is related to the inverse of the flatness of the potential, then physics that perturbs a flat direction in the potential would give this effect. In some supersymmetric models, loop corrections generate a logarithmic tilt $V \sim \phi^{n} \ln (\phi / \mu)[3,4]$. This would give $\Phi \approx \phi / n$ (or $\phi \ln (\phi / \mu)$ for $n=0$ ), and restricting $\phi<M_{P}$ (for the effective field theory to be valid), provides the limit $\Phi \lesssim \mathcal{O}\left(M_{P}\right)$. However, the generic breakdown of slow roll is independent of any $\Phi$ upper limit conjecture and we do not consider the latter further. Hierarchical parameters to replace slow roll are discussed in $\S \mathrm{VI}$.
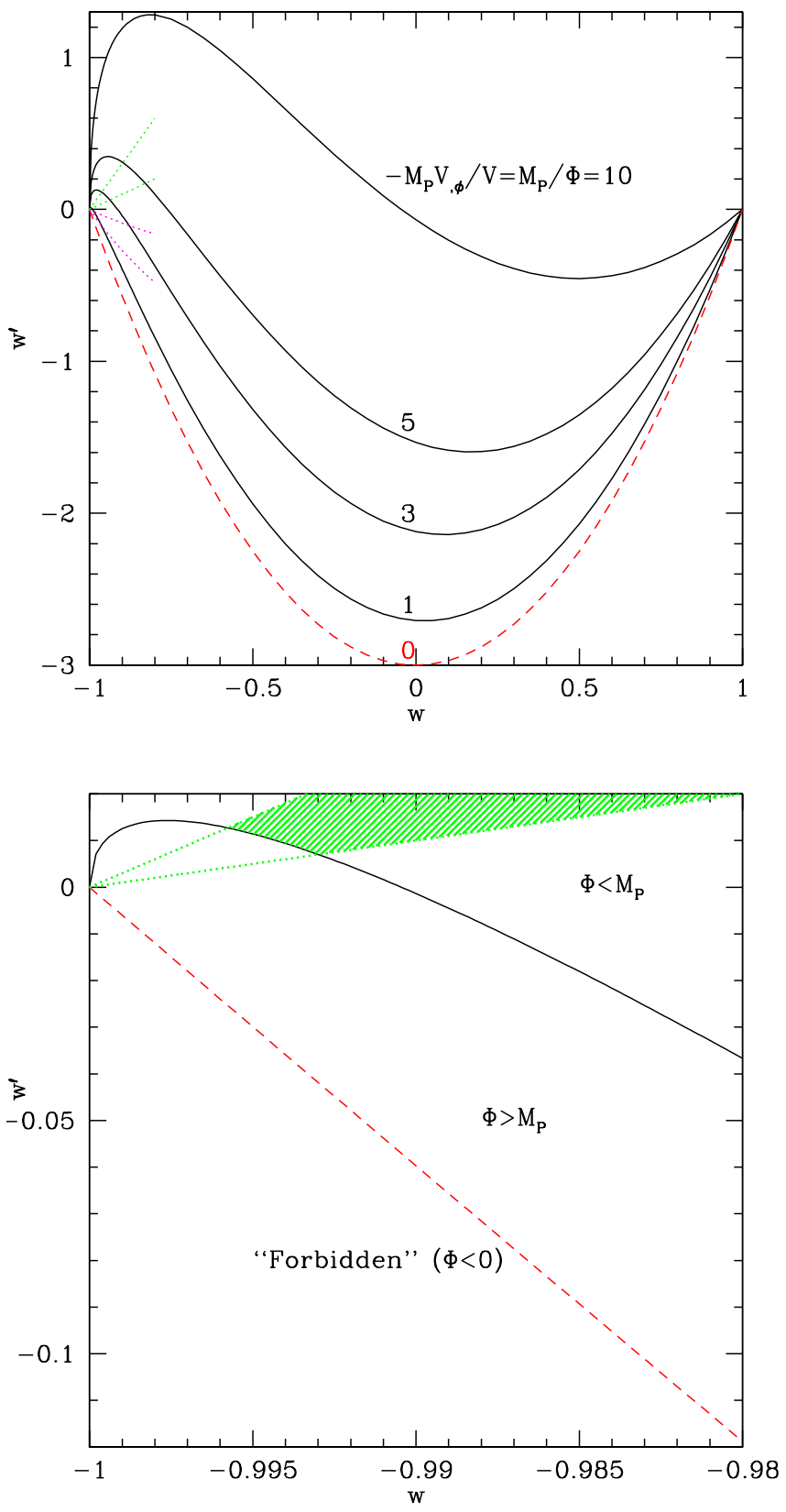

FIG. 1: Contours of the inverse of the characteristic field scale $\Phi=-V / V_{, \phi}$ are plotted in full and zoomed versions, for present matter density $\Omega_{m}=0.28$. Note that only a slender crescent of the phase plane obeys the conventional slow roll criterion $M_{P} / \Phi \ll 1$. The dashed red curve shows the null line $V_{, \phi}=0$. Dotted lines show the $w \approx-1$ limits of the thawing region (upper pair: $\left.w^{\prime}=3(1+w),(1+w)\right)$ and freezing region (lower pair: here $w^{\prime}=w(1+w), 3 w(1+w)$; not shown in bottom panel). If physics limits the characteristic field scale to be less than the Planck mass, then a barrier forms (second panel) around the cosmological constant, allowing only models in the shaded part of the thawing region. 


\section{B. Driving and dragging}

Returning to the Klein-Gordon equation, we can understand behavior in the $w-w^{\prime}$ phase space by first a general and then a specific analysis of the terms. Writing Eq. (2) as $\ddot{\phi}+3 H \dot{\phi}=-V_{, \phi}$, we can require $-V_{, \phi} \geq 0$, reflecting that the field rolls down the potential to large $\phi$. Using Eq. (4) for $\dot{\phi}$, we write this condition in terms of $w, w^{\prime}$ as $w^{\prime} \geq-3\left(1-w^{2}\right)$. The boundary defines the null line $V_{, \phi}=0$ discussed further below. Similarly, writing the Klein-Gordon equation as $\ddot{\phi}+V_{, \phi}=-3 H \dot{\phi}$ and again using that the field rolls to larger values with time implies $-3 H \dot{\phi} \leq 0$. This reduces to the condition $w \geq-1$.

If we flip the direction of the field motion and potential slope, i.e. the field rolls down the potential to smaller $\phi$, then these conditions remain. (I.e. $\dot{V}=\dot{\phi} V_{, \phi}$ is still negative, and so the transition from Eq. (5) to Eq. (6) flips the sign of the second term on the right hand side of Eq. (6), canceling the reversed sign of $V_{, \phi}$.) However, if we make the field move up the potential then we have the relations $w^{\prime} \leq-3\left(1-w^{2}\right)$ and $w<-1$, in the phantom region, i.e. the boundary lines just continue smoothly through $w=-1$.

Finally, we can move $\ddot{\phi}$ to the right hand side to obtain $3 H \dot{\phi}+V_{, \phi}=-\ddot{\phi}$. This divides the $w-w^{\prime}$ plane into regions where the field is accelerating or decelerating, with the boundary being the coasting behavior $\ddot{\phi}=0$. This line corresponds to the condition $w^{\prime}=3(1+w)^{2}$. Larger values of $w^{\prime}$ arise from a field accelerating down the potential, while smaller values come from a field decelerating (this motion of the field should not be confused with the accelerating expansion of the universe, which can hold for either region). These three boundaries - the null line $w^{\prime}=-3\left(1-w^{2}\right)$, coasting line $w^{\prime}=3(1+w)^{2}$, and phantom line $w=-1$ - define the broad characteristics of the phase plane.

To investigate the dynamics further, we must examine the dominance of the different terms in the equation of motion (2). The first term is the acceleration of the field, the second term a friction term, or Hubble drag, due to the expansion of the universe, and the third is a driving term due to the steepness of the potential. We define

$$
\begin{aligned}
X & =\frac{\ddot{\phi}}{H \dot{\phi}} \\
Y & =\frac{\ddot{\phi}}{V_{, \phi}} .
\end{aligned}
$$

Figure 2 shows curves of constant $X, Y$ in the $w-w^{\prime}$ plane. Note that $X=Y=0$ corresponds to an epoch of coasting in the scalar field dynamics, $\ddot{\phi}=0$, as discussed above. This is nongeneric, as the field would need to be finely tuned to neither accelerate due to the slope of the potential nor decelerate due to the Hubble drag, but be perfectly balanced. Indeed, the dynamics of scalar fields in Paper 1 avoid this region, causing the split into the distinct thawing and freezing regions, respectively above and below this line.

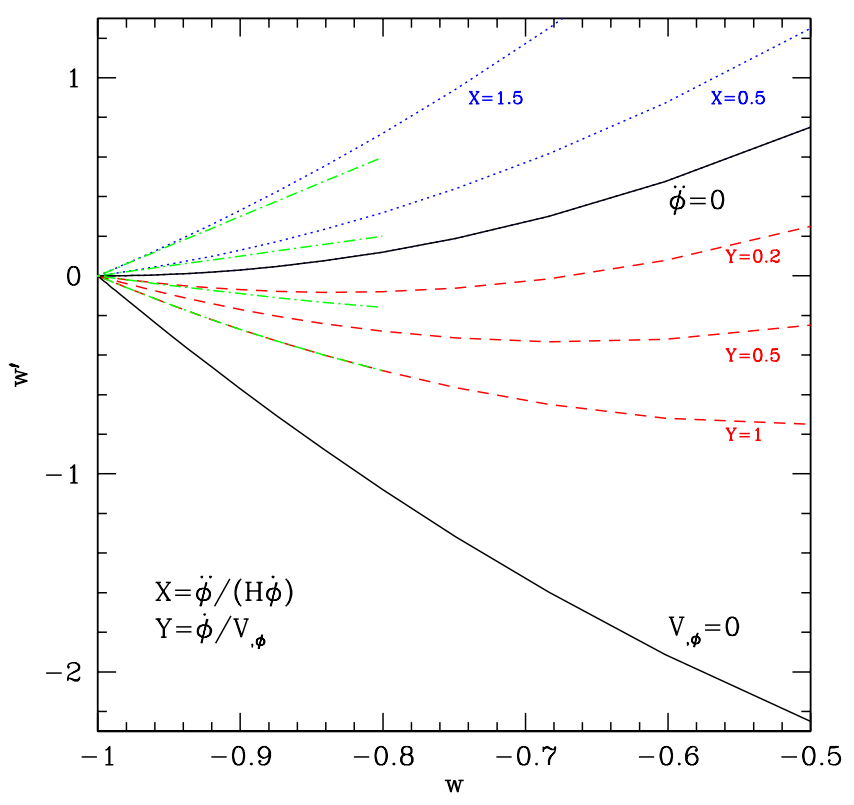

FIG. 2: The physics behind the dynamics becomes more apparent when looking at the ratios of the terms in the KleinGordon equation of motion, e.g. field acceleration vs. friction vs. potential driving. The key general features of the phase plane are the null line $V_{, \phi}=0$, the coasting line $\ddot{\phi}=0$, and the phantom line $w=-1$. Thawing (with $w^{\prime}>0$ ) and freezing (with $w^{\prime}<0$ ) regions are bounded by green, dot-dash curve pairs and are defined through the physical dynamics.

In the accelerating field region, the friction term is the major determinant of behavior initially, as the field evolves away from a frozen (cosmological constant-like) state in the matter dominated epoch. The upper boundary of the thawing region is given by $X=3 / 2$, where this value follows directly from the exponent of the expansion history, $t \sim a^{3 / 2}$ for matter domination. Thus fields that thaw during matter domination begin to move along the $X=3 / 2$ line (see discussion, and Figure 2, in Paper 1).

We can translate any acceleration to friction ratio $X$ to a $w-w^{\prime}$ behavior through

$$
\begin{aligned}
w^{\prime} & =2 X(1+w)+3(1+w)^{2}=(1+w)(3+2 X+3 w) \\
& \approx 2 X(1+w)
\end{aligned}
$$

where the last approximation is for $1+w \ll 1$. Note that the linear boundaries used in Paper 1 were (good) approximations to the general parabolic behavior. The value $X=0$ gives the coasting line and $X=-3$ gives the null line.

Thus, the upper thawing boundary $X=3 / 2$ corresponds to $w^{\prime} \approx 3(1+w)$ for $1+w \ll 1$. If the rolling field then enters a region where the potential slope is 
shallower (as usually happens), then the field will accelerate less and curve toward the $\ddot{\phi}=0$ line. Since today the field cannot have rolled so far that $\Omega_{\mathrm{de}}>0.8$, the dynamical track remains within the thawing region $1+w<w^{\prime}<3(1+w)$, i.e. $1 / 2<X<3 / 2$. For potentials that steepen as the field rolls down, e.g. PNGB models with the field starting near the top of the potential, the tracks instead lie above the $X=3 / 2$ or $w^{\prime}=3(1+w)$ line. The PNGB potential also steepens more rapidly for small symmetry scales $f$, and indeed, as mentioned in Linder [5], PNGB models roughly follow $w^{\prime}=F(1+w)$, where $F$ is proportional to the inverse of $f$. (Also see [6] for discussion of PNGB models, fine tuning, and slow roll.)

In the decelerating field region, the steepness of the potential impacts the freezing. As the potential becomes shallower, the friction is more effective. In the limit of a flat potential, one obtains the dynamics track given by the null curve, $V_{, \phi}=0$, in Fig. 2. As given in Paper 1 , this corresponds to $w^{\prime}=-3\left(1-w^{2}\right)$, and the skating model of $[7,8]$. In terms of the friction and driving terms, $X=-3$ and $Y=\infty$. The lower boundary of the freezing region lies along the $Y=1(X=-3 / 2)$ line, equivalent to $w^{\prime}=3 w(1+w)$. (See $\S$ VIB for a rationale.) We will later see that this line also has physical significance.

The general relation between the acceleration to steepness ratio $Y$ and the $w-w^{\prime}$ track is

$w^{\prime}=3(1+w)\left[w+\frac{1-Y}{1+Y}\right]=3 w(1+w)+3(1+w) \frac{1-Y}{1+Y}$

Again we have a parabolic behavior. The coasting line has $Y=0$, and the null line corresponds to $Y \rightarrow \infty$. One could use either variable $X$ or $Y$ throughout the phase plane, since

$$
X=-3 \frac{Y}{1+Y} \quad ; \quad Y=-\frac{X}{X+3}
$$

but this somewhat obscures the physics of friction and steepness. (That said, we note that the thawing/freezing boundaries are fairly symmetric in $X$, with the outer boundaries at $X= \pm 3 / 2$ and the inner ones at $X \approx$ $\pm 1 / 2.)^{1}$

Note that it is not legitimate to assume tracking behavior (where the equation of state is constant and related to the dominant component's equation of state) to impose limits on regions in the phase plane, as for example $[9,10]$ did to try to tighten the constraints of Paper 1. For one thing, not all freezing models need start as trackers. Secondly, just because the most negative value of $w^{\prime}$ lies above some boundary curve does not ensure that the entire trajectory remains above the curve. Most

\footnotetext{
${ }^{1}$ Recall from Paper 1 that the upper bound on the freezing region is not sharply defined, and extends somewhat above the $w^{\prime}=$ $w(1+w)$ line shown for convenience in this paper.
}

importantly, the tracking approximation breaks down as the dark energy first becomes significant, so it is inapplicable for much of the observable dynamical history.

\section{MORE SPECIFIC DYNAMICS}

In addition to analyzing general behavior through the Klein-Gordon equation terms, we can investigate the properties of the phase plane or specific track families in terms of other variables. These could include working from the equation of state $w-w^{\prime}$ relation directly or the cosmic expansion history $a(t)$. While not quite as insightful as the physics motivated driving and drag terms, they can highlight interesting properties.

\section{A. Mocker models}

Consider a model with dynamics given by $w^{\prime}=C w(1+$ $w)$. Note that while such an equation forms the boundaries of the freezing region, freezing models do not follow such a trajectory but rather are almost orthogonal to such tracks (at least initially). So we are talking about fundamentally different models. The behavior of the dark energy equation of state and density follow

$$
\begin{aligned}
w(a) & =-1+\left[1-\frac{w_{0}}{1+w_{0}} a^{C}\right]^{-1} \\
\rho_{\mathrm{de}}(a) & =\rho_{\mathrm{de}}(1)\left[\left(1+w_{0}\right) a^{-C}-w_{0}\right]^{3 / C},
\end{aligned}
$$

where $w_{0}$ is the equation of state today.

In the past, $a \ll 1$, the component will act like additional nonrelativistic matter, with $w=0$, while in the future it will approach a cosmological constant. Since such dark energy sometimes looks like dark matter and sometimes like a cosmological constant, we call this a "mocker" model. These are basically what are known as "quartessence" models (see [11] for an overview), of which the Chaplygin gas is one example. However, we develop them directly from the phase space dynamics rather than an ansatz for the pressure, so the dynamical behavior is more general. For example, a constant pressure model could be a cosmological constant, or could be a mocker with $C=3$. Note $C=3$ gives precisely the expression for the lower boundary of the freezing region (or is $Y=1$ or $X=-3 / 2$ in the notation of $\S$ II B). We name the $w^{\prime}=3 w(1+w)$ curve the constant pressure line (also see [10] in the context of barotropic fluids).

Such combined behavior models are heir to all the usual problems of trying to unify dark matter and dark energy, e.g. growth instabilities of density perturbations $[12,13]$. Analysis of perturbations requires knowledge of the full theory, however. Merely from the phase plane dynamics, though, we can see trouble arising for such unified models. As $C$ gets smaller, the model moves along its trajectory more quickly, acting less like dark matter except at very early times, $1+z \gg\left[-w_{0} /\left(1+w_{0}\right)\right]^{1 / C}$. 
Conversely, as $C$ gets larger, acceleration of the cosmic expansion occurs later and the model becomes a poorer fit to a host of (purely geometric) observations such as supernova distances and the distance to the CMB last scattering surface.

\section{B. Relation to parameterized $w(a)$}

The approach taken in this article is to examine dark energy dynamics directly in the phase plane $w-w^{\prime}$, where a time variable runs along each trajectory. It is useful to see the relation of standard parametrizations in terms of the temporal behavior, i.e. $w(a)$, to this approach.

The standard two parameter function $w(a)=w_{0}+$ $w_{a}(1-a)$ was shown by Linder $[14,15]$ to provide an excellent approximation to exact solutions of the KleinGordon equation in a wide variety of models. In this ansatz we have $w^{\prime}=-a w_{a}=w-w_{\infty}$, where the high redshift equation of state $w_{\infty}=w_{0}+w_{a}$. This describes a straight line of slope 1 in the $w-w^{\prime}$ plane, and can be rewritten as as $w^{\prime}=(1+w)-\left(1+w_{\infty}\right)$. In particular, if $w_{\infty}=-1$ we have exactly the behavior of thawing models (lying along lower bound of that region). From Fig. 2 of Paper 1, we see as well that many tracking models that fit present data (i.e. $\Omega_{\text {de }} \sim 0.7$ and $w<$ -0.8 ) are reasonably well described, on average, by a line of slope unity. Of course this approximation will break down in the future, as the field freezes more fully, turning toward the cosmological constant; at the same time this $w(a)$ ansatz loses validity as it moves toward ever more negative $w$. However, since data only exist toward the past, we see why the $w_{a}$ parametrization is an excellent approximation.

To retain boundedness for both the past and present, as well as to allow more dramatic dynamics (essentially slopes other than unity in $w-w^{\prime}$ ), one could use the "efold" model of [16] or "kink" model of [17]. Both utilize four parameters for their description. The e-fold model has a more transparent translation to $w^{\prime}=d w / d \ln a$ since it also uses dynamics in terms of $\ln a$ :

$$
w(a)=w_{f}+\frac{\Delta w}{1+\left(a / a_{t}\right)^{\tau}},
$$

where $\tau$ is the transition rapidity in units of units of e-folds $\ln a, a_{t}$ is the transition scale factor, $w_{f}$ is the asymptotic future value, and $\Delta w=w_{p}-w_{f}$ is the difference between asymptotic past and future values.

In the $w-w^{\prime}$ phase plane we have

$$
w^{\prime}=-\tau\left(w-w_{f}\right)\left(1-\frac{w-w_{f}}{\Delta w}\right) .
$$

Note that as we found in the Klein-Gordon equation analysis of $\S \mathrm{IIB}$ the equation for $w^{\prime}$ is quadratic in $w$. We can identify several special cases. If the asymptotic future state is deSitter $\left(w_{f}=-1\right)$, then $w^{\prime}=$ $\tau\left(\Delta w^{-1}-1\right)(1+w)+(\tau / \Delta w) w(1+w)$. This looks like the sum of a thawing model and a model in the freezing region, i.e. the dark energy can be viewed as the sum of two components. If we further take $\Delta w=1$, then we remove the thawing component and end up with $w^{\prime}=\tau w(1+w)$ - a mocker model with $w_{p}=0$ and $w_{f}=-1$.

Starting instead with an asymptotic past state of $w_{p}=$ -1 gives $w^{\prime}=\tau(1+w)\left(w-w_{f}\right) /\left(-1-w_{f}\right)$. In the limit $w_{f} \rightarrow \infty$ (i.e. not worrying about the region where there is no data) this gives a thawing model $w^{\prime}=\tau(1+$ $w)$. Thus the four parameter e-fold ansatz is also quite versatile. The rapidity parameter is directly related to both the slope of and the velocity along the phase space trajectory, and ties in with the steepness of the scalar field potential, as we saw in $\S I I B$ with the PNGB models where the slope was proportional to the inverse of the symmetry scale $f$.

Finally, one could invert the situation and go from a parametrization in the phase plane to derive the function $w(a)$. For example, a track $w^{\prime}=A(1+w)+B(1+w)^{2}$, which we have seen is a common form, implies

$$
1+w=\left(1+w_{0}\right) /\left[(1+x) a^{-A}-x\right],
$$

where $x=(B / A)\left(1+w_{0}\right)$ defines the present along the trajectory (equivalently the dimensionless matter density $\Omega_{m}$ today). Note that while the trajectory has two parameters, the equation of state has three parameters since we must have a parameter running along the track. At high redshift, if $A>0$ then $w \rightarrow-1$ and we have a thawing model, asymptotically independent of $B$. If $A<0$ then $w(z \gg 1)=-1+(-A / B)$ and $w^{\prime} \rightarrow 0$, i.e. it begins like a tracking model. It reaches a minimum $w_{\min }^{\prime}=$ $-A^{2} /(4 B)$ at $w_{\star}=-1-A /(2 B)=[-1+w(z \gg 1)] / 2$; that is, the trajectory is a parabola from its tracking value of the equation of state to its future, cosmological constant value. A mocker model is the special case $C=-A=B$. For completeness, we give the dark energy density:

$$
\rho_{\mathrm{de}}(a)=\rho_{\mathrm{de}}(1)\left(1+x-x a^{A}\right)^{3 / B} .
$$

Of particular interest is the "leveling" model where, in loose physical analogy to the inflationary power spectrum tilt $n-1$ being driven to zero by large numbers of efolds of expansion, the equation of state tilt $1+w$ is driven to zero by the deSitter expansion as the energy density approaches a certain constant value, $\rho_{f}$. That is, take $1+w=D\left[\rho_{\mathrm{de}}(a)-\rho_{f}\right]$. This is equivalent to the above parabolic model with $A=-3 D \rho_{f}$ and $B=-3$. Another interesting parabolic track is the coasting line $w^{\prime}=3(1+w)^{2}$. This corresponds to not a leveling but a tilting, with $1+w=\left(1+w_{0}\right)\left[\rho_{\mathrm{de}}(1) / \rho_{\mathrm{de}}(a)\right]$, so $w$ is tilted away from -1 as the energy density decreases.

\section{Acceleration and jerk}

One could leave behind the physics of the accelerating phenomenon and instead use variables in terms of the 
acceleration itself, though this seems less appealing. The deceleration parameter

$$
q=-a \ddot{a} / \dot{a}^{2}=\frac{1}{2}+\frac{3}{2} w \Omega_{d e}(a)
$$

and the jerk

$$
j=a^{2} \dddot{a} / \dot{a}^{3}=1-\frac{3}{2} \Omega_{d e}(a)\left[w^{\prime}-3 w(1+w)\right] .
$$

We also have $j=q+2 q^{2}-q^{\prime}$. Note that interpreting $q$ and $j$ or $q^{\prime}$ as Taylor expansions of the expansion is of strictly limited use (since observations span $\Delta \ln a \sim \mathcal{O}(1)$ ) and can be dangerous [18].

Furthermore, there is the same ambiguity there was with using pressure as a variable. We note that any model where it touches the constant pressure line $w^{\prime}=$ $3 w(1+w)$ has $j=1$; this is equivalent to $X=-3 / 2$ in the notation of $\S$ II B. The two standard special cases of $j=1$ lie at the ends of this line: an Einstein-de Sitter universe with $w=0$ "dark energy" and a $\Lambda$ CDM universe with cosmological constant dark energy.

The constant pressure line is also related to the adiabatic sound speed of the dark energy. (Note this is not the true sound speed of perturbations arising from the microphysics of whatever the dark energy is.) The adiabatic sound speed

$$
c_{a}^{2}=\frac{\dot{p}}{\dot{\rho}}=w-\frac{1}{3} \frac{w^{\prime}}{1+w},
$$

and we see it vanishes for $w^{\prime}=3 w(1+w)$. On the null line, the adiabatic sound speed equals the speed of light (the same as the true sound speed for a canonical scalar field). Models below the null line would need to have $c_{a}^{2}>1$.

\section{BEYOND SCALAR FIELDS}

For the cosmic expansion dynamics we can always define an effective equation of state even if the accelerating mechanism is not a scalar field [1], through

$$
w_{\mathrm{eff}}=-1-\frac{1}{3} \frac{d \ln \delta H^{2}}{d \ln a}
$$

where $\delta H^{2}=\left(H / H_{0}\right)^{2}-\Omega_{m} a^{-3}$ is the unknown part of the Hubble parameter, that not due to matter. So it is of interest to see to what extent the dynamical behaviors we have discussed carry over to the $w_{\text {eff }}-w_{\text {eff }}^{\prime}$ plane. That is, are freezing and thawing behaviors more general than for scalar fields, and do the null and coasting lines still play a role?

Due to the diversity of possible accelerating physics we do not present a general analysis of these important questions but rather calculate some specific cases.

\section{A. Scalar-tensor gravity}

Scalar-tensor theories modify the Einstein-Hilbert action with both an additional scalar field and a coupling to Ricci scalar curvature $R$. These are of great interest as a comparison in tests of general relativity and also because gravitational theories involving a nontrivial function of the scalar curvature can be transformed to scalar-tensor theories. See [19] for a general introduction.

We consider coupling of a general form in the scalar field, but linear in the curvature. So the general relativistic $R /(8 \pi G) \rightarrow F(\phi) R$. One then obtains the usual Friedmann expansion equations, with extra terms giving an effective dark energy density [20]

$\rho_{\mathrm{ST}}=V(\phi)+(1 / 2) H^{2}(q-1)(q+5) F_{\phi}^{2}+3 H^{2}\left[(8 \pi G)^{-1}-F\right]$,

where $q$ is the deceleration parameter, $F_{\phi}=d F / d \phi$, and the last term involves the change of the gravitational strength from Newton's constant $G$.

From this density one can then define $\delta H^{2}=$ $8 \pi G \rho_{\mathrm{ST}} /\left(3 H^{2}\right)$ (note that we use the usual $G$ here since the deviation is absorbed into $\rho_{\mathrm{ST}}$ as mentioned above). From this the equation of state $w_{\text {eff }}$ and its variation $w_{\text {eff }}^{\prime}$ can be calculated. A key quantity will be $F / F_{\phi}^{2} \equiv \omega_{J B D}$. This is the Jordan-Brans-Dicke parameter and its inverse must be very small according to solar system tests. Expanding $F$ about the present,

$$
\begin{aligned}
F(a) & \approx(8 \pi G)^{-1}-(1-a) F_{\phi} \dot{\phi} /(a H) \\
& \approx(8 \pi G)^{-1}-z(1-q) F_{\phi}^{2}
\end{aligned}
$$

So the ratio of the second to the first term $\left(\sim \omega_{J B D}^{-1}\right)$ is small, and gravity is nearly Einsteinian.

But this means that the first term in $\rho_{\mathrm{ST}}$ dominates (unless $8 \pi G V / H^{2} \ll 1$, but then it doesn't affect the expansion and there is no acceleration). Thus, the restriction of the scalar-tensor theory by solar system constraints means that its effective equation of state must be very close to a cosmological constant - within $\sim \omega_{J B D}^{-1}$. Since $\omega_{J B D}^{-1}<2.5 \times 10^{-5}$, this would be rather challenging to distinguish from a cosmological constant with cosmological observations! One possible loophole is if the solar system limits on the scalar coupling should not be applied to a cosmological situation because of the different spacetime backgrounds with very different scalar curvatures. This arises for example in chameleon scenarios [21]. The most stringent cosmological bounds on varying $G$ arise from primordial nucleosynthesis and give $\omega_{J B D}^{-1} \lesssim 3 \times 10^{-3}[22]$ (but see [23]).

Calculation of the effective phase plane parameters finds [24]

$$
\begin{aligned}
& w_{\mathrm{eff}}(z=0)=-1+0.46 / \omega_{J B D} \\
& w_{\mathrm{eff}}^{\prime}(z=0)=-0.36 / \omega_{J B D} .
\end{aligned}
$$

While even with only the cosmological bounds on $\omega_{J B D}$ these are quite close to the cosmological constant in the 
phase plane, it is interesting to note that the current values lie along $w^{\prime}=0.78 w(1+w)$, in the freezing region. Indeed its trajectory is a freezing one, with scalartensor theories asymptotically attracted to general relativity $[25,26]$, and to $w=-1$. One last thing to note, however, is that because scalar-tensor theories possess anisotropic stress, the growth of density perturbations will be modified from the quintessence case (see, e.g., [27] and references therein).

\section{B. Braneworld cosmology and $H^{\alpha}$}

In a braneworld cosmology [28, 29], effective acceleration appears due to a weakening of gravity on large scales as it "leaks" from our brane into a higher dimensional bulk. The Friedmann expansion equation becomes

$$
H^{2}-H / r_{c}=8 \pi G \rho_{m} / 3
$$

where $r_{c}$ is the crossover distance and $\rho_{m}$ the matter density. The effective equation of state due to the modification is $w_{\text {eff }}=-\left[1+\Omega_{m}(a)\right]^{-1}[30]$. Its trajectory in the phase plane is plotted in Fig. 3. Note that it looks like a freezing model, and will indeed approach a cosmological constant in the asymptotic future.

The position along the trajectory is a time variable, so taking the present to be, say, $\Omega_{m}=0.2$ would extend the solid curve in Fig. 3 slightly further (since the figure uses $\left.\Omega_{m}=0.3\right)$. We also see why it is so well approximated by a $w_{0}-w_{a}$ model, as discussed in $\S I I I B$. (Recall, however, that $w_{a}$ is actually defined at $z=1$, not $z=0$, to give the best physics fit [14].)

We can further generalize the modification to $\delta H^{2} \sim$ $H^{\alpha}[31]$. Then we find

$$
\begin{aligned}
& w_{\mathrm{eff}}=-\left[1-\frac{\alpha}{\alpha-2} \Omega_{m}(a)\right]^{-1} \\
& w_{\mathrm{eff}}^{\prime}=3 w(1+w)[1-(2 / \alpha)(1+w)] .
\end{aligned}
$$

Recall that the braneworld case above corresponds to $\alpha=1$. For acceleration today (with $\Omega_{m}=0.3$ ), we require $\alpha<1.57$; for $w<-0.8$ today we require $\alpha<$ 0.91. Note that all $H^{\alpha}$ modified gravity models will look similar (one does require $\alpha<2$ for a negative equation of state at early times). See $\S$ VIB for discussion of their tracking behavior. In particular, they all approach the cosmological constant along $w^{\prime}=3 w(1+w)$, what was called the constant pressure line for scalar fields. Their tracks must always lie between $w^{\prime}=3 w(1+w)$ and the $w^{\prime}=0$ axis. When $\alpha<0$, the trajectories switch to the phantom regime with $w<-1$, but the bounds still hold.

\section{POLYTROPIC DARK ENERGY}

An interesting, if phenomenological, way of obtaining acceleration is to modify the Friedmann expansion

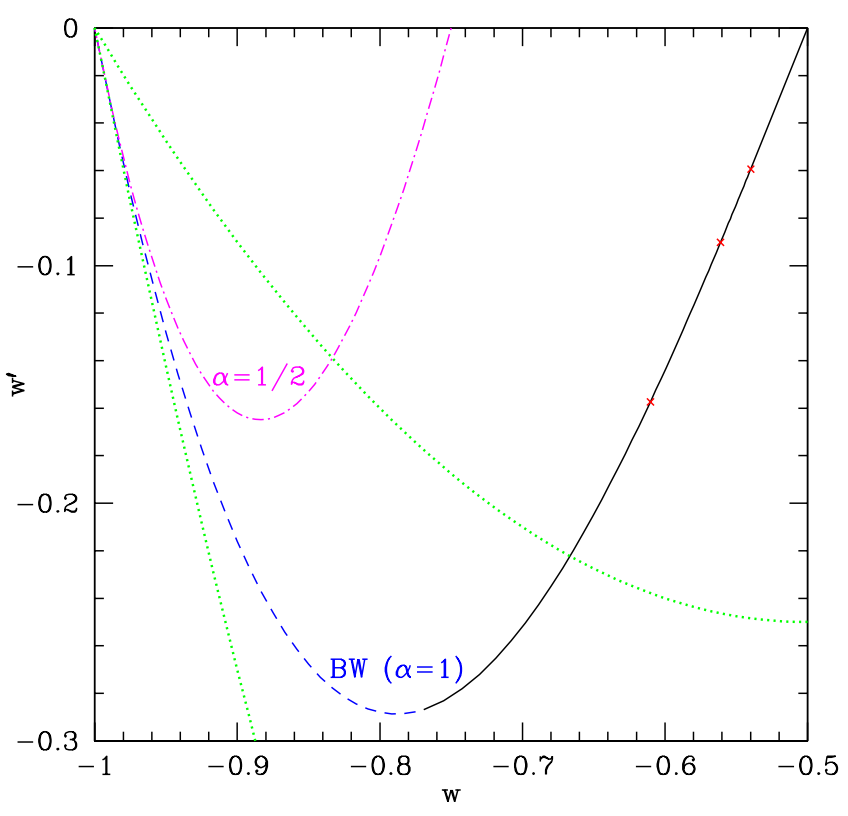

FIG. 3: The braneworld model of modified gravity behaves like a freezing scalar field. The solid black line shows its trajectory: starting at high redshift at $w=-1 / 2, w^{\prime}=0$, with the red crosses indicating $z=3,2,1$ before the line ends at the present. The dashed blue line shows its future behavior, approaching a cosmological constant, and lying within the quintessence freezing bounds of $w^{\prime}=3 w(1+w)$ and $w^{\prime}=$ $w(1+w)$ given by the dotted green curves. A model with a generalized, extra $H^{\alpha}$ term (for the braneworld, $\alpha=1$ ) in the Friedmann expansion equation looks similar; the magenta, dot-dash curve is for $\alpha=0.5$.

equation but keeping a pure matter universe. While this leaves open important questions about its relation to fundamental theory and the growth of density perturbations, we can investigate some general aspects of the effective equation of state dynamics.

Consider general functions of the matter density (sometimes referred to as barotropic models [10])

$$
H^{2}=(8 \pi G / 3) g(\rho)=(8 \pi G / 3)[\rho+f(\rho)] .
$$

The quantity $f(\rho)$ will act like an effective dark energy. Using Eq. (22) we can define

$$
\begin{aligned}
& w_{\mathrm{eff}}=-1+\frac{d \ln f}{d \ln \rho} \\
& w_{\mathrm{eff}}^{\prime}=-3 \frac{d^{2} \ln f}{d \ln \rho^{2}}
\end{aligned}
$$

and identical relations hold for the total equation of state $w_{\text {tot }}$ and its variation $w_{\text {tot }}^{\prime}$ upon substituting $g$ for $f$.

The simplest example of such a modification is $f \sim \rho^{n}$, the Cardassian model of [32]. From the above equations we see that it corresponds to a constant equation of state $w=-1+n$. If we require $w<-0.9$ (as observations 
favor for a constant equation of state), then $n<0.1$; unfortunately $\rho^{1 / 10}$ does not obviously appear to be a natural modification of the Friedmann equation.

We can investigate the phase space dynamics by relating $w^{\prime}$ to $w$ :

$$
w^{\prime}=3 w(1+w)-3 \frac{\rho^{2}}{f} \frac{d^{2} f}{d \rho^{2}} .
$$

Immediately we see that whether the effective dark energy lies below the freezing region or not depends on the sign of $d^{2} f / d \rho^{2}$. An equivalent question is whether the effective pressure is decreasing or increasing with time (since the $w^{\prime}=3 w(1+w)$ line is that of constant pressure).

The model will follow the mocker model track $w^{\prime}=$ $3 w(1+w)$ if $f=A+B \rho$. This form is equivalent to a redefinition of $\Omega_{m}$, e.g. $\Omega_{m} \rightarrow \Omega_{m}(1+B)$, plus a cosmological constant $A$. As such it has a nonzero minimum in its effective potential, allowing it to reach the freezing boundary.

It is important to remember that the analysis in this paper and Paper 1 applies to the dynamics of the dark energy itself. Trajectories in the $w_{\text {tot }}-w_{\text {tot }}^{\prime}$ plane convolve the matter and dark energy components, mixing the dynamics and so not giving rise to the clear differentiations and regions found. This is why Ref. [10] appears to find a violation of the freezing bound and even null bound for some barotropic models; those are actually phantom models in the dark energy phase space, but are dragged by the matter to $w_{\text {tot }}>-1$. We make this more explicit later in this section.

For a richer dynamical behavior we propose a class of modifications of the Friedmann expansion equation we call polytropic models [33]. Here

$$
H^{2} /(8 \pi G / 3)=g(\rho)=\rho\left[1+\left(\rho / \rho_{\star}\right)^{-\alpha}\right]^{\beta} .
$$

At densities much greater than some crossover value $\rho_{\star}$, e.g. at high redshift, the Friedmann equation is standard. At low densities, the expansion is modified, with $w_{\text {tot }}$ asymptotically approaching $-\alpha \beta$. If we want a future deSitter state, we could choose $\beta=1 / \alpha$. For just the effective dark energy equation of state, the value in the past is $w_{\text {eff }}=-\alpha$, and in the future of course it dominates so $w_{\text {eff }}=-\alpha \beta$.

Figure 4 illustrates the phase plane dynamics. The first panel takes $\beta=1 / \alpha$, so that the future state is deSitter. Models with $\alpha<1$ have $w \geq-1$ and act like freezing models, starting from a constant $w=-\alpha$ and today (marked by crosses) lying in the freezing region, before heading toward the cosmological constant. Phantom models have $\alpha>1$ and act like mirror images of freezing models, even to lying within the phantom freezing region today.

In the second panel, we fix $\beta=1 / 2$. While the models start at the same phase space point as the previous models with the same $\alpha$, now their endpoints are different. Indeed for $\alpha<2 / 3$ the acceleration of the expansion is
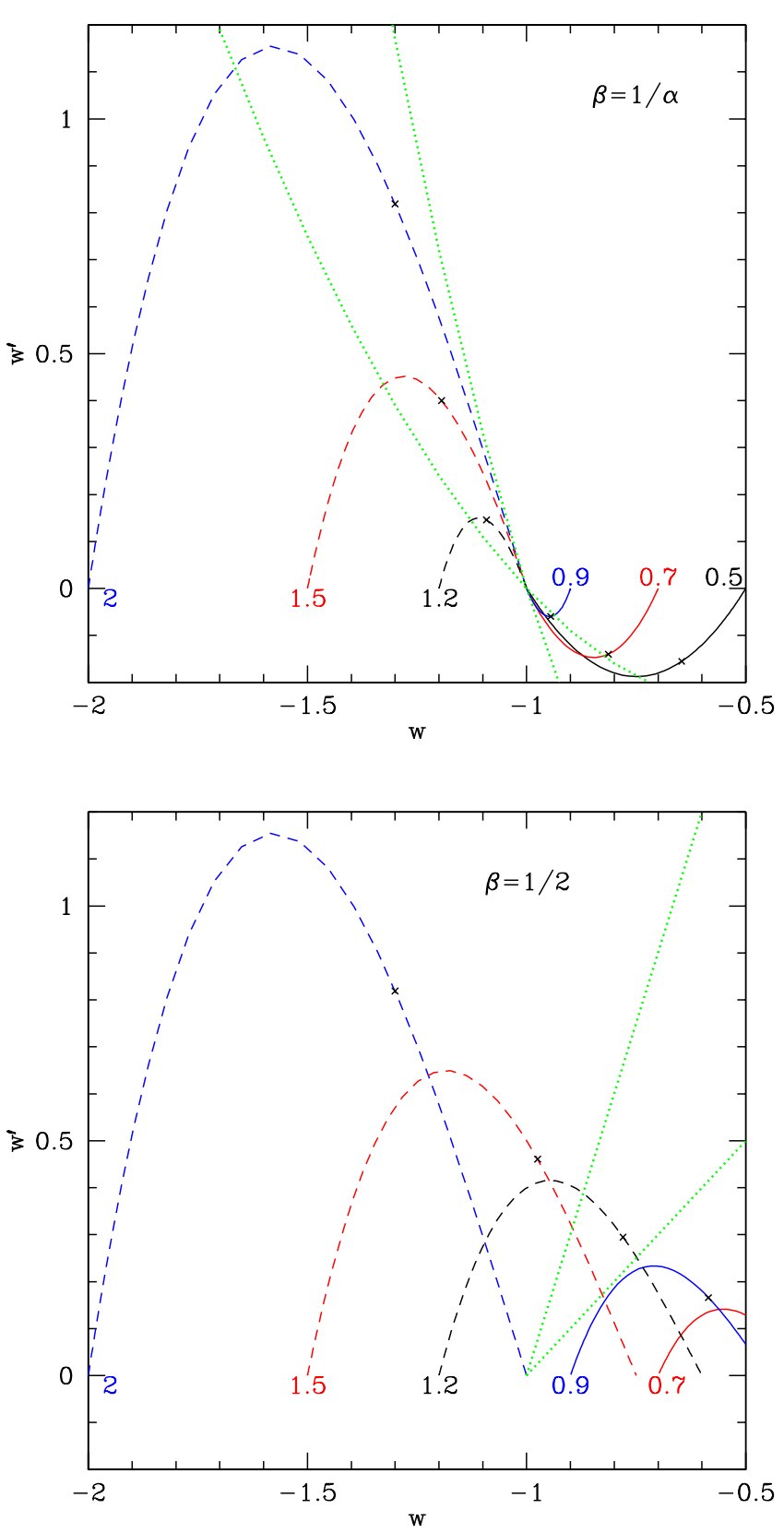

FIG. 4: Polytropic models, with $\beta=1 / \alpha$ in the first panel and with $\beta=1 / 2$ in the second panel. Curves are labeled with $\alpha$ and crosses show the present. Dotted curves show the canonical scalar field freezing region (first panel) or thawing region (second panel). In the first panel, models evolve toward a cosmological constant, within the freezing region. In the second panel, the asymptotic state is not deSitter (except for the $\alpha=2$ curve, which is the same in both panels) and the dynamics is distinct from a canonical scalar field.

a temporary phase. Furthermore, the trajectories with $\alpha \lesssim 1.5$ do not lie in the freezing regime and both regular and phantom models have $w^{\prime}>0$. Such polytropic 
models without a deSitter future will be clearly distinguishable from both freezing and thawing quintessence.

Can we give some physics motivation for the polytropic form, aside from its simplicity and proper asymptotic behavior? When $\beta=0$, there is no modification; when $\beta=1$ we have the power law modification of the Cardassian case, with $n=1-\alpha$, hence a constant $w=-\alpha$. There are some motivations for power law modification from higher dimension theories (for $n<1$ see [32], the nonaccelerating $n=2$ arises in Randall-Sundrum brane scenarios [34]). When $\beta=1 / 2$, the modification is similar to that from a Chaplygin gas [35], as we see below; this has claimed motivation from Born-Infeld actions and brane solutions [36]. So at least the polytropic form unifies different prescriptions for dark energy.

Note that as $\beta$ increases from zero, for fixed $\alpha$, the size of the "hump" in the trajectory decreases and the future value of $w$ moves back toward the initial value. At $\beta=1$ the trajectory collapses to a point at $w=-\alpha, w^{\prime}=0$. For even larger $\beta$, the hump is flipped (i.e. the sign of $w^{\prime}$ changes) and again increases in size, with the future value of $w$ drawing away to more negative values.

When we plot the same models as in the first panel of Fig. 4 , but in the $w_{\text {tot }}-w_{\text {tot }}^{\prime}$ plane, in Fig. 5 , we see that the models that were phantom in the effective dark energy lie in the region $w_{\text {tot }}^{\prime}<3 w_{\text {tot }}\left(1+w_{\text {tot }}\right)$. Furthermore, when $\alpha>2$ they can even lie below what was the null line $w^{\prime}=-3\left(1-w^{2}\right)$. In a nice analysis of barotropic models, Scherrer [10] noted something similar (his barotropic models are a function of an arbitrary component density not necessarily matter density). For a barotropic perfect fluid the adiabatic sound speed is the physically relevant sound speed for perturbations (but not in the quintessence case, or in a general multicomponent case), and Scherrer's bound of $w^{\prime}<3 w(1+w)$ holds for $c_{a}^{2}>0$ (cf. Eq. 21 here). In general, however, this is not a violation of the bounds of this article and Paper 1 because it occurs only when the adiabatic assumption holds, e.g. when viewing the total equation of state, not the properties of a (non-adiabatic) effective dark energy.

Suppose, however, we chose to fit the dark energy component itself, rather than full energy density entering the Friedmann equation, by the polytropic form Eq. (35). This is somewhat strange to do, since then the effective dark energy contains a matter-like part, in addition to the pure matter density, and the polytrope was designed to be the modified Friedmann equation as a whole. If we do so, though, then the dark energy equation of state phase plane (and not the total equation of state) is represented by the curves in Fig. 5. Moreover, the curve with $\alpha=2, \beta=1 / 2$ is the trajectory of the Chaplygin gas. The generalized Chaplygin gas with pressure $p \sim-\rho^{-\alpha_{g c g}}$ corresponds to polytropic dark energy with $\alpha=\alpha_{g c g}+1, \beta=1 / \alpha$. Whenever $\alpha \beta=1$ (cf. [37]) we have mocker behavior with $w^{\prime}=3 \alpha w(1+w)$. As stated above, however, taking the dark energy itself to be polytropic means hiding both a cosmological constant (if $\beta=1 / \alpha$ ) and a spurious matter density within the

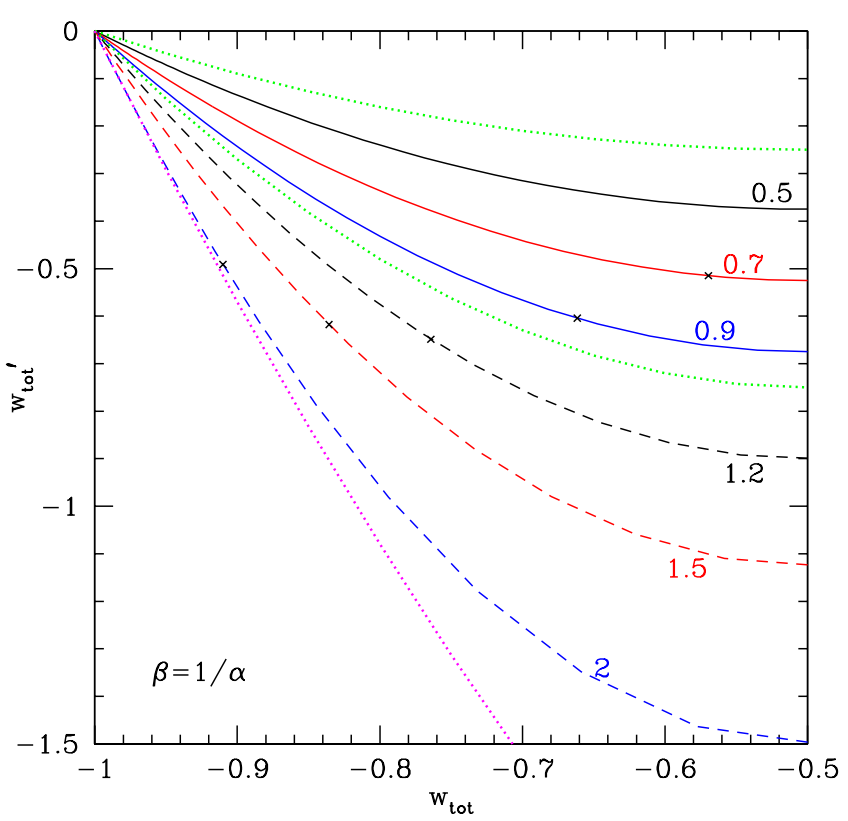

FIG. 5: Polytropic models as Fig. 4a, with $\beta=1 / \alpha$, but now viewed in the total equation of state phase plane. Curves are labeled with $\alpha$ and crosses show the present. Dotted curves show the dark energy freezing region bounds (light green) and the null line (dark magenta). Note that those models phantom in the dark energy here appear with $w_{\text {tot }}>-1$ but lie below $w_{\text {tot }}^{\prime}=3 w_{\text {tot }}\left(1+w_{\text {tot }}\right)$. One should not apply the dark energy dynamics constraints to the total equation of state plane.

dark sector.

\section{HIERARCHY PARAMETERS}

Returning to more general dynamics, we saw in $\S \mathrm{II}$ and in particular from Fig. 1 that we do not have the standard inflation slow roll perturbative expansion parameter. Explicitly, we do not have small $V_{,_{\phi}} / V$ in the freezing or thawing regions unless $w \lesssim-0.995$; more generally, $-M_{P} V_{, \phi} / V \gtrsim 5 \sqrt{1+w}$ today. We examine here whether we can substitute a physics based hierarchy of dynamics parameters.

\section{A. Slope parameters}

The Klein-Gordon equation can be rewritten dimensionlessly as

$$
\phi^{\prime \prime}+(2-q) \phi^{\prime}=-V_{, \phi} / H^{2} \equiv \eta_{1},
$$

where as before a prime denotes a derivative with respect to $\ln a$, and $q=-a \ddot{a} / \dot{a}^{2}$ is the deceleration parameter. In terms of the $w-w^{\prime}$ dynamics equation,

$$
w^{\prime}=-3\left(1-w^{2}\right)-\sqrt{2\left(1-w^{2}\right)} V_{, \phi} /\left(H V^{1 / 2}\right)
$$




$$
\equiv-3\left(1-w^{2}\right)+\sqrt{2\left(1-w^{2}\right)} \eta_{2}
$$

So the parameters $\eta_{1}, \eta_{2}$ are called out by the physics. If they are small, one could solve the equations perturbatively. Note that $\eta_{2}=\eta_{1}\left(V / H^{2}\right)^{-1 / 2}$ so we always have $\eta_{2}>\eta_{1}$. We violate the lower bound of the freezing region, $w^{\prime}<3 w(1+w)$, when

$$
\eta_{2}^{2}<\frac{9}{2} \frac{\epsilon}{2-\epsilon}
$$

where $\epsilon=1+w$ is the tilt parameter. The analogous condition such that $w^{\prime}<0$ is $\eta_{2}^{2}<(9 / 2) \epsilon(2-\epsilon)$, and such that field is decelerating $(\ddot{\phi}<0)$ is $\eta_{2}^{2}<18 \epsilon /(2-\epsilon)$.

Neither $\eta_{1}$ nor $\eta_{2}$ are particularly small unless $\epsilon=1+w$ is. For example $\eta_{2} \gtrsim 1.5 \sqrt{\epsilon}$, giving $\eta_{2}>0.1$ for $w>$ -0.995 . Even $\eta_{1}>0.1$ for $w>-0.94$. The hierarchy among the parameters is fixed for the region of interest: $\epsilon<\eta_{1}<\eta_{2}$, so there is no phase space classification in this respect, as there is for large field, small field, and hybrid models in inflation [38]. However that hierarchy involved the second derivative of the potential, so it is worth a brief look at that quantity.

\section{B. Tracking parameter}

The tracking parameter is defined to be

$$
\Gamma \equiv \frac{V V_{, \phi \phi}}{V_{, \phi}^{2}}
$$

This is not generally a small parameter either. Indeed, models whose energy density tracks [39] the evolution of the dominant energy component fulfill the conditions

$$
\Gamma>1 \quad ; \quad \frac{d \ln (\Gamma-1)}{d \ln a} \ll 1
$$

Within the class of tracking models (so now a particular subset of scalar field cosmologies), at high redshifts within the matter dominated epoch the field obeys

$$
\Gamma=1-\frac{w}{2(1+w)} .
$$

(Note the equation of state deviation $1+w$ at high redshift may not be small.)

In $\S I V B$ we found that $H^{\alpha}$ models (including braneworlds) follow freezing trajectories. This is not surprising because they are basically trackers. A component starting with constant $w$ at early times is equivalent there to a modification $H^{\alpha}$ with $\alpha=2(1+w)$. The tracking parameter $\Gamma=(2+\alpha) /(2 \alpha)$ and it initially acts like an inverse power law potential $V \sim \phi^{-n}$ with $n=2 \alpha /(2-\alpha)$.

To relate the dynamics of the time variation to the tracking condition, we invert Eq. (42) to write

$$
\begin{aligned}
w & =-2(\Gamma-1) /[1+2(\Gamma-1)] \\
w^{\prime} & =\frac{d w}{d \ln a}=w(1+w) \frac{d \ln (\Gamma-1)}{d \ln a} .
\end{aligned}
$$

However, this is of limited use since we are unlikely to be able to probe $w^{\prime}$ in the high redshift, $z \gg 1$, regime where tracking might hold. Strong acceleration today, with $w \lesssim-0.7$, requires the breakdown of tracking.

However, the analogy to $H^{\alpha}$ models presents an important insight into why the general freezing region is bounded below by $w^{\prime}=3 w(1+w)$. At early times the contribution to the Friedmann expansion equation by the dark energy is small and $\delta H^{2} \sim a^{-3(1+w)} \sim H^{2(1+w)}$. That is, the effective $\alpha \approx 2(1+w)$. One can generalize this to $\alpha=2\langle 1+w\rangle$ when time variation of the equation of state becomes relevant, where angle brackets denote an averaging over $\ln a$. At late times, the dark energy density dominates the expansion, $\delta H^{2} \sim H^{2}$, as it approaches $w=-1$ (freezes). For any epoch we can define an instantaneous value of $\alpha$. Equation (30) then gives the relation for $w^{\prime}$. As freezing models approach $w=-1$, Eq. (30) indicates they should do so along $w^{\prime}=3 w(1+w)$. Furthermore, since the bracketed term is less than one, then this trajectory represents a general lower bound to the freezing region. ${ }^{2}$

\section{Dynamics, Mass, and Spatial Inhomogeneities}

Dynamical models must also possess spatial inhomogeneities in the field at some level. The equation for these is given by perturbation of the Klein-Gordon equation (2),

$$
\delta \ddot{\phi}+3 H \delta \dot{\phi}+\left(k^{2}+V_{, \phi \phi}\right) \delta \phi=-\dot{h} \dot{\phi} / 2,
$$

where $k$ is the wavenumber and $h$ is the trace of the metric perturbation [40]. Just as matter density perturbations are damped on scales below the Jeans length related to the sound speed in the background medium, so the spatial inhomogeneities in the scalar field will be absent on length scales less than that corresponding to the effective mass $\sqrt{V_{, \phi \phi}}$.

Using eq. (6) and $V_{, \phi \phi}=\dot{V}_{, \phi} / \dot{\phi}$ we can calculate the critical mass scale (also see [41]), with

$$
\begin{aligned}
V_{, \phi \phi} / H^{2}= & (2+3 w+2 q) \frac{w^{\prime}}{1+w}+\frac{1}{4}\left(\frac{w^{\prime}}{1+w}\right)^{2} \\
& -\frac{1}{2} \frac{w^{\prime \prime}}{1+w}+\frac{3}{4}(1-w)(5+3 w+2 q)
\end{aligned}
$$

Note $w^{\prime} /(1+w)$ and $w^{\prime \prime} /(1+w)$ are well behaved and generally nonzero as $w \rightarrow-1$.

This shows that the goal of exploring the temporal and spatial dynamics of dark energy runs into double jeopardy. If the time variation is weak, $\left|w^{\prime} /(1+w)\right| \ll 1$,

\footnotetext{
2 One caveat involves dark energy models that possess an internal cosmological constant, i.e. nonzero minimum to the potential, or otherwise act as the sum of two or more components. These cannot be represented as $H^{\alpha}$ models and the freezing bound does not apply.
} 
then the effective mass $m=\sqrt{V_{, \phi \phi}} \lesssim H$. (The scale $H$ today corresponds to $10^{-33} \mathrm{eV}$; dark energy would be a very light scalar field). This means the Compton wavelength of the scalar field perturbations is larger than the horizon, and so spatial inhomogeneities are also difficult to detect. Thus, for $\left|w^{\prime}\right|<1+w$, i.e. between the thawing and freezing regions there is a "dead zone" of phase space, where we can detect neither time variation nor spatial inhomogeneity.

For appreciable time variation, $\left|w^{\prime}\right|>1+w$, one can have $m>H$ and so the possibility of subhorizon clustering. However for models within the freezing or thawing regions, one is restricted to $m \lesssim 2 H$ so this could only occur on the largest scales (largest angles or lowest multipoles). Note that as the field approaches $w=-1$, the mass stays nonzero (except it vanishes along the upper boundary of thawing and along the null line). However, the amplitude of spatial perturbations vanishes as can be seen from Eq. (45) with $\dot{\phi}=0$. These properties make scalar field inhomogeneity an extremely begrudging probe of the nature of dark energy, much less friendly than the dynamics.

\section{CONCLUSION}

We have deepened and elaborated the understanding of the role that the dark energy dynamics, through the $w-w^{\prime}$ phase plane, can play in leading our understanding of the nature of dark energy. This includes the foundations of the null line, coasting line, constant pressure line, and phantom line dividing the phase plane into distinct, physical regions. We also elucidate the uppermost and lowermost boundaries of the thawing and freezing regions.

The physical structure has been extended beyond canonical scalar fields, including specific instances of modified gravity scenarios such as scalar-tensor, braneworld, and $H^{\alpha}$ models, and barotropic and polytropic generalizations of the Friedmann equation. We outlined similarities and differences with the scalar field case, showing that many act as freezing fields, and that we should be able to clearly distinguish certain models that do not possess a deSitter future. Mocker models, im- plementing a unification of dark matter and dark energy, were shown to have difficulties purely from dynamical considerations, in addition to their problems in structure formation.

Dark energy is demonstrated to be generically not amenable to a slow roll description - a major difference from early universe inflation - as one of its "Goldilocks" conundra. This makes the dark energy problem in some sense even more challenging than the early universe. However, it also opens the possibility that if some physical bound can be placed on the flatness of the potential, e.g. due to quantum corrections, then this implies a barrier around the cosmological constant $\Lambda$ model. This would offer hope, possibly accessible to next generation experiments, that dark energy could definitely be distinguished from $\Lambda$, if it is not $\Lambda$. That would be exciting!

The dynamics of the dark energy, in the form of the equation of state ratio $w$ and its time variation $w^{\prime}$, provides powerful insight into the new physics behind cosmic acceleration. Spatial inhomogeneities in the dark energy are seen to be much weaker and less forthcoming, unless one entertains direct couplings. While we are not guaranteed to zero in on the physics - there is a dead zone of minimal dynamics and possibly a "confusion" zone near the cosmological constant - any highly precise and accurate result would be an enormous success in enlightening us on the dark universe.

\section{Acknowledgments}

I benefited greatly from numerous discussions with my collaborator Robert Caldwell. I also thank Carlo Baccigalupi, Robert Scherrer, and participants in the workshop Cosmological Frontiers in Fundamental Physics at Perimeter Institute (which I thank for hospitality), the workshop Dark Energy from Fundamentals (DarkFun 3, hosted by the SNAP Collaboration), and the LBNL particle theory group. This work has been supported in part by the Director, Office of Science, Department of Energy under grant DE-AC02-05CH11231.
[1] E.V. Linder \& A. Jenkins, MNRAS 346, 573 (2003) [astro-ph/0305286]

[2] R.R. Caldwell \& E.V. Linder, Phys. Rev. Lett. 95, 141301 (2005) [astro-ph/0505494]

[3] E. Witten, Phys. Lett. B 105, 267 (1981)

[4] T. Damour \& V.F. Mukhanov, Phys. Rev. Lett. 80, 3440 (1998) [gr-qc/9712061]

[5] E.V. Linder, Astropart. Phys. 25, 167 (2005) [astro$\mathrm{ph} / 0511415]$

[6] N. Kaloper \& L. Sorbo, astro-ph/0511543

[7] E.V. Linder, Astropart. Phys. 24, 391 (2005) [astro- $\mathrm{ph} / 0508333]$

[8] M. Sahlén, A.R. Liddle, D. Parkinson, Phys. Rev. D 72, 083511 (2005) [astro-ph/0506696]

[9] T. Chiba, astro-ph/0510598

[10] R.J. Scherrer, Phys. Rev. D 73, 043502 (2006) [astro$\mathrm{ph} / 0509890]$

[11] M. Makler, S.Q. de Oliviera, I. Waga, Phys. Rev. D 68, 123521 (2003) [astro-ph/0306507]

[12] H. Sandvik, M. Tegmark, M. Zaldarriaga, I. Waga, Phys. Rev. D 69, 123524 (2004) [astro-ph/0212114]

[13] L. Amendola, I. Waga, F. Finelli, JCAP 0511, 009 (2005) 
[astro-ph/0509099]

[14] E.V. Linder, Phys. Rev. Lett. 90, 091301 (2003) [astro$\mathrm{ph} / 0208512]$

[15] E.V. Linder, in Identification of Dark Matter (IDM2002), eds. N.J.C. Spooner \& V. Kudryavtsev (World Scientific: 2003) [astro-ph/0210217]

[16] E.V. Linder \& D. Huterer, Phys. Rev. D 72, 043509 (2005) [astro-ph/0505330]

[17] P.-S. Corasaniti \& E.J. Copeland, Phys. Rev. D 67, 063521 (2003) [astro-ph/0205544]

[18] J.-M. Virey et al., Phys. Rev. D 72, 061302 (2005) [astro$\mathrm{ph} / 0502163]$

[19] Y. Fujii \& K. Maeda, The Scalar-Tensor Theory of Gravitation (Cambridge U. Press: 2003)

[20] G. Esposito-Farèse \& D. Polarski, Phys. Rev. D 63, 063504 (2001) [gr-qc/0009034]; F. Perrotta \& C. Baccigalupi, Phys. Rev. D 65, 123505 (2002) [astroph/0201335]

[21] P. Brax et al., Phys. Rev. D 70, 123518 (2004) [astro$\mathrm{ph} / 0408415]$

[22] T. Clifton, J.D. Barrow, R.J. Scherrer, Phys. Rev. D 71, 123526 (2005) [astro-ph/0504418]

[23] A. de Felice, G. Mangano, M. Trodden, astro-ph/0510359

[24] C. Baccigalupi, E.V. Linder, S. Matarrese, F. Perrotta, in preparation

[25] T. Damour \& B. Pichon, Phys. Rev. D 59, 123502 (1999) [astro-ph $/ 9807176]$

[26] S. Matarrese, C. Baccigalupi, F. Perrotta, Phys. Rev. D 70, 061301 (2004) [astro-ph/0403480]

[27] F. Perrotta, S. Matarrese, M. Pietroni, C. Schimd, Phys. Rev. D 69, 084004 (2004) [astro-ph/0310359]; V. Acquaviva, C. Baccigalupi, F. Perrotta, Phys. Rev. D 70, 023515 (2004) [astro-ph/0403654]
[28] G. Dvali, G. Gabadadze, M. Porrati, Phys. Lett. B 485, 208 (2000) [hep-th/0005016]

[29] C. Deffayet, G. Dvali, G. Gabadadze, Phys. Rev. D 65, 044023 (2002) [astro-ph/0105068]

[30] A. Lue, R. Scoccimarro, G.D. Starkman, Phys. Rev. D 69, 124015 (2004) [astro-ph/0401515]

[31] G. Dvali \& M.S. Turner, astro-ph/0301510

[32] K. Freese \& M. Lewis, Phys. Lett. B 540, 1 (2002) [astro$\mathrm{ph} / 0201229]$

[33] After this article was finished, we found earlier mention of this form in: K. Freese, Nucl. Phys. B 124, 50 (2003) [hep-ph/0208264]

[34] L. Randall \& R. Sundrum, Phys. Lett. B 83, 4690 (1999) [hep-th/9906064]

[35] A. Kamenshchik, U. Moschella, V. Pasquier, Phys. Lett. B 511, 265 (2001) [gr-qc/0103004]

[36] M.C. Bento, O. Bertolami, A.A. Sen, Phys. Rev. D 66, 043507 (2002) [gr-qc/0202064]

[37] P. Gondolo \& K. Freese, Phys. Rev. D 68, 063509 (2003) [hep-ph/0209322]

[38] W.H. Kinney, E.W. Kolb, A. Melchiorri, A. Riotto, Phys. Rev. D 69, 103516 (2004) [hep-ph/03050130]

[39] I. Zlatev, L. Wang, P.J. Steinhardt, Phys. Rev. Lett. 82, 896 (1999) [astro-ph/9807002]

[40] C.-P. Ma, R.R. Caldwell, P. Bode, L. Wang, Ap. J. Lett. 521, L1 (1999) [astro-ph/9906174]; R.R. Caldwell, R. Dave, P.J. Steinhardt, Phys. Rev. Lett. 80, 1582 (1998) [astro-ph/9708069]

[41] R.R. Caldwell, in Sources and Detection of Dark Matter in the Universe (DM2000), ed. D. Cline, p. 74 (Springer: 2001); http://www.dartmouth.edu/ caldwell/index_files/DM2000.ps 